Christine Dörner · Klaudia Erhardt (Hrsg.)

Politische Meinungsbildung und Wahlverhalten 
Schriften des Zentralinstituts für sozialwissenschaftliche Forschung der Freien Universität Berlin

ehemals Schriften des Instituts für politische Wissenschaft

Band 84 
Christine Dörner · Klaudia Erhardt (Hrsg.)

\section{Politische Meinungsbildung und Wahlverhalten}

Analysen zum „Superwabljabr“ 1994

Westdeutscher Verlag 
Die Deutsche Bibliothek - CIP-Einheitsaufnahme

Politische Meinungsbildung und Wahlverhalten : Analysen zum „Superwahljahr“ 1994 / Christine Dörner ; Klaudia Ehrhardt (Hrsg.). Opladen ; Wiesbaden : Westdt. Verl., 1998

(Schriften des Zentralinstituts für Sozialwissenschaftliche Forschung der Freien Universität Berlin ; Bd. 84)

ISBN-13: 978-3-531-13273-0 e-ISBN-13: 978-3-322-89876-0

DOI: $10.1007 / 978-3-322-89876-0$

Alle Rechte vorbehalten

(C) Westdeutscher Verlag GmbH, Opladen/Wiesbaden, 1998

Der Westdeutsche Verlag ist ein Unternehmen der Bertelsmann Fachinformation GmbH.

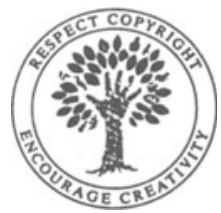

Das Werk einschließlich aller seiner Teile ist urheberrechtlich geschützt. Jede Verwertung außerhalb der engen Grenzen des Urheberrechtsgesetzes ist ohne Zustimmung des Verlags unzulässig und strafbar. Das gilt insbesondere für Vervielfältigungen, Übersetzungen, Mikroverfilmungen und die Einspeicherung und Verarbeitung in elektronischen Systemen.

http://www.westdeutschervlg.de

Höchste inhaltliche und technische Qualität unserer Produkte ist unser Ziel. Bei der Produktion und Verbreitung unserer Bücher wollen wir die Umwelt schonen: Dieses Buch ist auf säurefreiem und chlorfrei gebleichtem Papier gedruckt. Die Einschweißfolie besteht aus Polyäthylen und damit aus organischen Grundstoffen, die weder bei der Herstellung noch bei der Verbrennung Schadstoffe freisetzen.

Umschlaggestaltung: Christine Huth, Wiesbaden 


\section{Inhalt}

Christine Dörner/Klaudia Erhardt

Einführung

Klaudia Erhardt

Die unentschlossenen Wähler als Motor zyklischer Wahlabsichtsverläufe. Eine Untersuchung am Beispiel der Rückgewinnung der Wähler durch die CDU im Frühjahr 1994

Christine Dörner

Do Media Matter? - Zur Reichweite des Agenda Settings

Christine Dörner

Die Wählerschaft von Bündnis 90/Die Grünen - demographische Zusammensetzung und politische Orientierung

Ivo Scheike

Die Wählerschaft der Republikaner: Eine Analyse vor dem Hintergrund ihres früheren Wahlverhaltens

Wolfram Schulz

Die Kanzlerkandidaten Kohl und Scharping in der Medien-

berichterstattung des Wahljahrs 1994

Korrespondenzanschriften der Autoren 


\section{Vorwort}

Die in diesem Band vorgelegten Beiträge sind Arbeitsergebnisse des von der Deutschen Forschungsgemeinschaft geförderten Forschungsprojekts "Wahlabstinenz, Wählerabwanderung und politische Issues. Eine Längsschnittstudie zu Ursachen und Wirkungen politischer Problemthematisierung im 'Superwahljahr' 1994", kurz auch als Projekt "Massenmedien und Wähler" bezeichnet. Es handelte sich um ein interdisziplinäres Forschungsprojekt, an dem Wissenschaftler der Fachbereiche Politische Wissenschaft und Publizistik der Freien Universität Berlin sowie des Wissenschaftszentrums Berlin beteiligt waren. Zusammen mit dem kürzlich veröffentlichten Band von Richard Stöss ${ }^{1}$ liegen hiermit die wesentlichen Ergebnisse der Projektarbeitsgruppe am Fachbereich Politische Wissenschaft vor.

Wir danken der Deutschen Forschungsgemeinschaft, die das Projekt drei Jahre lang gefördert hat. Weiterhin danken wir Manfred Güllner, dem Leiter von FORSA, für die großzügige Überlassung von Befragungsdaten, die den ursprünglichen Auftragsumfang erheblich überschritten haben, Frauke Burian für ihre Geduld und das sorgfältige Lektorat und nicht zuletzt all jenen, die das Entstehen der vorliegenden Beiträge mit Anregungen und sachkundiger Kritik unterstützt haben.

Berlin, im Januar 1998

Die Herausgeberinnen

1 Richard Stöss, Stabilität im Umbruch. Wahlbeständigkeit und Parteienwettbewerb im "Superwahljahr" 1994, Opladen 1997. 